\title{
Recent advances on graphical evaluation and review techniques
}

\author{
Mohammad Reza Ghaeli $^{{ }^{*}}$ and Soheil Sadi-Nezhad
}

${ }^{a}$ Adjunct Professor, School of Management, New York Institute of Technology, 1700 - 701 W Georgia St., Vancouver, BC V7Y 1 K8 Canada ${ }^{b}$ Department of Finance and Statistics, University of Waterloo, Waterloo, Canada

\section{CHR O N I CLE A B S T RACT}

Article history:

Received: March 5, 2017

Received in revised format: July

16,2017

Accepted: July 20, 2017

Available online:

July 20, 2017

Keywords:

GERT

Graphical evaluation and review

techniques

Network

\begin{abstract}
Graphical evaluation and review techniques (GERT) is a technique to study the stochastic nature of networks consisting of different branches. In GERT, all branches are explained in terms of the probability that the branch is traversed and the tile to traverse the branch in case it is realized. This paper presents recent advances of the implementation of GERT in various industries. The study presents a comprehensive description of GERT and recent advances on the implementation of GERT in various industries over the period 2002-2017.
\end{abstract}

\section{Introduction}

Networks and network analysis play essential role for description and improvement of different systems due to simplification of modeling techniques (Pritsker \& Whitehouse, 1966). This makes it possible for analyzing the complicated systems in a less inductive way and could stimulate tasks in the network analysis. This also gives a comprehensive breakthrough to simplify the development of analysis procedure for more complex networks (FriLòker, 1966; Chen \& Bao, 2013).

Graphical Evaluation and Review Techniques (GERT) is a technique to study the stochastic nature of networks consisting of different branches. In GERT, all branches are explained in terms of the probability that the branch is traversed and the tile to traverse the branch in case it is taken (FriLòker, 1966; Li et al., 2012; Wu et al., 2014; Hu et al., 2010). Sridharan and Kalyani (2002), for instance, presented a stochastic analysis of a non-identical two-unit parallel system with common-cause failure based on the GERT technique.

* Corresponding author.

E-mail address: rghaeli@nyit.edu (M.R. Ghaeli)

2017 Growing Science Ltd. doi: $10.5267 / j . j p m .2017 .7 .001$ 


\subsection{Components of stochastic network}

A stochastic network consists of directed branches including arcs, edges and transmittances. It also includes logical nodes or vertices. Let $\mathrm{P}_{\mathrm{a}}$ be the probability that the node from which it emanated is realized. Also, let $t_{a}$ be the required time for accomplishment of the task in case the branch is taken. The following shows the visual representation of a directed branch.

$$
\left(\mathrm{P}_{\mathrm{a}} ; \mathrm{t}_{\mathrm{a}}\right)
$$

A node in a stochastic network includes of an input and an output. Under such circumstance, three logical relations on the input part and two kinds of relations on the output side may appear as depicted in Table 1.

Table 1

Descriptions of the nodes (FriLòker, 1966)

\begin{tabular}{ll}
\hline Exclusive-or & $\begin{array}{l}\text { Description } \\
\text { The realization of any branch leading into the node } \\
\text { causes the node to be realized; however, one and } \\
\text { only one of the branches leading into this node can } \\
\text { be realized at a particular time. }\end{array}$ \\
Inclusive-or & $\begin{array}{l}\text { The realization of any branch leading into the node } \\
\text { causes the node to be realized. The time of realiza- } \\
\text { tion is the smallest of the completion times of the ac- } \\
\text { tivities leading into the Inclusive-Or node. }\end{array}$ \\
And & $\begin{array}{l}\text { The nodes is realized only if the branches leading } \\
\text { into the node are realized. The time of the realization } \\
\text { therefore is determined by the largest completion } \\
\text { time. }\end{array}$ \\
Probabilistic & $\begin{array}{l}\text { All branches emanating from the node are taken if } \\
\text { the node is realized. }\end{array}$ \\
Exactly one branch emanating from the node is \\
taken if the node is realized.
\end{tabular}

\subsection{A simple example}

Consider a sales mission where two salesman have to successfully offer some products. In order for the sales to be completed successfully, both salesman must sell their products. The stochastic network for this problem is shown in Fig. 1 as follows,

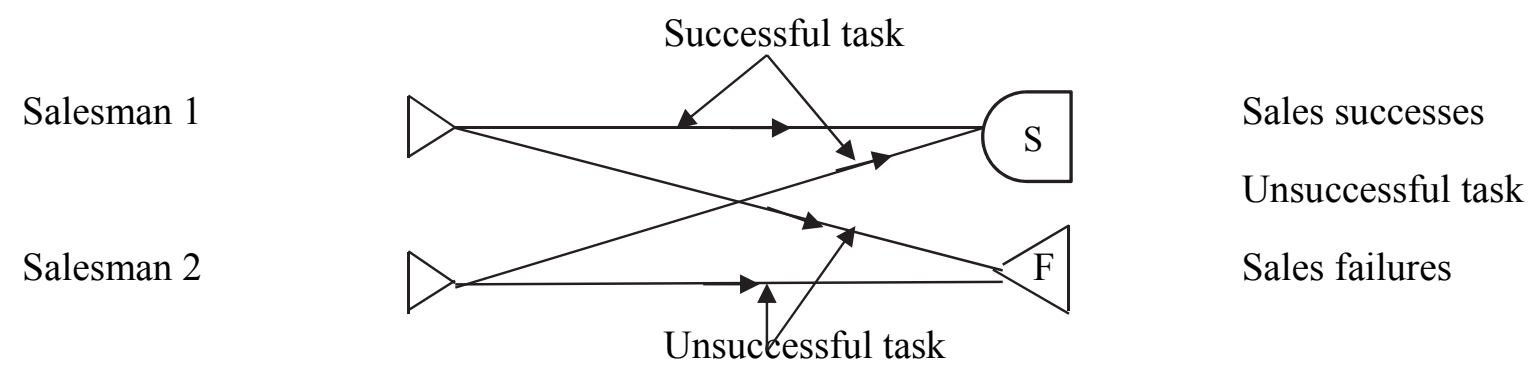

Fig. 1. A simple GERT prototype 
For the node $\mathrm{S}$ to be realized, both branches leading into it must be realized, which represents a characteristics of the AND node. In addition, $\mathrm{F}$ is realized only if either branch incident to it is realized, which represents an Inclusive-Or node.

GERT has been used for solving many complicated problems. In this paper, we present a comprehensive review on recent advances of the applications of GERT in different industries.

\section{GERT applications}

$\mathrm{CO} 2$ emission has become a serious problem in today's environmental science and many people have attempted to find ways for $\mathrm{CO} 2$ emission reduction through different research and development projects. Kosugi et al. (2004) presented an application of GERT for the success of research and development projects in reducing CO2 emission. In this study, GERT-type network charts were built to make an assessment on the R\&D processes of five kinds of $\mathrm{CO} 2$ capture technologies having various levels of CO2 capture energy efficiency. To do so, they collected the necessary data on R\&D time periods of different elemental technologies through a questionnaire to experts. GERT models were used for making assessments on the $\mathrm{CO} 2$ capture technology $\mathrm{R} \& \mathrm{Ds}$, and estimating the overall R\&D times and the success probabilities for realizing the target technologies. Hayashi et al. (2005) also investigated polymer electrolyte fuel cell application technology R\&Ds by GERT analysis. Guo and \& Qiao (2004) proposed a modeling method of product development process by applying GERT to optimize process model in product R\&D under resource constraints. They built a hierarchical GERT network to explain the product development process. The activity-time parameters in the process were also measured.

Yamashiro (2008) analyzed some network models using GERT systems. Mohan et al. (2009) and Agarwal et al. (2007) used GERT for analysis of some reliability problems. They studied consecutivek-out-of-n-out-of-:F systems with sparse d, m-consecutive-k-out-of-n:F systems with sparse d, and (n, $\mathrm{f}, \mathrm{k})$ :F systems with sparse d.

Yi-song et al. (2009) analyzed the logical relationship of WBS items by applying the GERT network description of WBS items of a product development project and used it in product development process.

$\mathrm{Xu}$ and Jia (2010) proposed the use of the failure probability and multi-branch feedback of the weapon system into a GERT network in order to prevent over-cost and time delay in the weapon system development. They also built a feedback simulation model based on the GERT network including multibranch.

Li et al. (2011) presented a GERT-based analytical technique for uncertainty management of remanufacturing. They first proposed four GERT-based stochastic Remanufacturing Process Routing (RPR) models and analyzed a remanufacturing system that consists of different process flows, where the probability of and the time associated with individual process were measured.

Lin et al. (2011a,b,c) developed a weakest t-norm $\left(\mathbf{T}_{\mathbf{\omega}}\right)$ fuzzy GERT simulation technology, which was designed to be useful in a realistic environment and to improve based on the traditional fuzzy GERT insofar as was developed for analyzing complex systems in uncertain environments; the traditional system usually adopts $\boldsymbol{\alpha}$-cut arithmetic operations for its calculations. In this study, the fuzzy support system developed the $\mathbf{T}_{\boldsymbol{\omega}}$ fuzzy GERT as a substitute for traditional fuzzy GERT technology. In the simple cases investigated, the fuzzy support system built a model of $300 \mathrm{~mm}$ manufacturing processes in the context of a lithography area. In addition, the manufacturing processes model was investigated with regard to the fuzzy support system based on two kinds of fuzzy arithmetic: $\boldsymbol{\alpha}$-cut arithmetic and the $\mathbf{T}_{\boldsymbol{\omega}}$ operator. 
Wang et al. (2011) presented a vague GERT for assessing wafer manufacturing yield and finishing time in lithographic area. They proposed a simple decision support system to process the complex calculation procedure for enhancing more information to managers. They also incorporated the capability of lithographic area in order to improve overall system performance.

Li et al. (2014) investigated the cost distribution characteristics in multi-stage supply chain networks. They proposed a stochastic network mathematical model for cost distribution analysis in multi-stage supply chain networks based on GERT method. In addition, to study the impacts of cost components, including the procurement, inventory, shortage, production and transportation costs of supply chain members, on the total supply chain operation cost, they proposed the concept of cost sensitivity and provided corresponding algorithms based on the proposed stochastic network model. They also extended the model to analyze the cost performance of supply chain robustness under various order compensation ability scenarios and the corresponding algorithms were developed.

Gangeshwer and Shankar (2015) presented GERT analysis of the deferred life test sampling plan based on gamma failure. GERT approach which has been successfully used by Ohta (1978) for investigating some quality control problems. The idea of applying the deferred sampling plan goes back to Vaerst (1980). This procedure gave a visual picture of the dynamics of the inspection system and also offered a through characterization of the plan.

Zhou et al. (2016) presented a forecasting model which could forecast the quantity, time and probability of product return, as well as recyclable parts/components/materials and disposal using GERT by translating the remanufacturing operational process into a stochastic network. The stochastic network possessed two characteristics: 1) activities having a probability of occurrence associated with them; and 2) time to perform an activity. They also used a numerical example to show the result of using GERT on forecasting printer remanufacturing outcomes.

Nelson et al. (2016) presented a time-computing model using GERT to analyze concurrent New Product Development (NPD) processes. They first conceptualized a concurrent NPD process based on the GERT scheduling technique and extracted a method of modelling the information and communication complexities within the process. Next, they extended previous research carried out on concurrent engineering and included it within our model. They also presented an alternative method of analyzing concurrent NPD process for both researchers and project managers alike.

Tao et al. (2017) introduced a GERT model based on a characteristic function and designed its numerical solution. First, they implemented an inversion formula to extract the probability distribution of the completion time of a product development. Next, to investigate the applications of a due date, they introduced a novel measure of schedule risk to provide a view of both loss and probability. Last, they used an elasticity analysis to determine the network parameters that expedite the control of schedule risk. They also presented a case study of new product development in a high-technology enterprise to show the implementation of their proposed methods.

Liu et al. (2017) presented an analytical algorithm for the GERT network model to receive the value transfer probability, the amount of value increment, and the fluctuation variance, from which the equivalence value transfer process. They also implemented the proposed method and realized that a value transfer relationship within the carbon fiber industry chain in Jiangsu Province, China.

\section{Conclusion}

As we can see from the review process provided in this survey, there are literally different fields of studies to investigate the industrial applications of GERT, from research and development to $\mathrm{CO} 2$ emission prevention and from reliability problems to redundancy applications. The survey has indicated that researches mostly use GERT when they face with more complicated problems. 


\section{Acknowledgement}

The authors would like to thank the anonymous referees for constructive comments on earlier version of this paper.

\section{References}

Agarwal, M., Sen, K., \& Mohan, P. (2007). GERT analysis of $m$-consecutive-k-out-of- $n$ systems. IEEE Transactions on Reliability, 56(1), 26-34.

Chen, T. H., \& Bao, C. P. (2013). Applying cost-reliability analysis to improve system reliability. Journal of Industrial and Production Engineering, 30(7), 467-472.

FriLòker, A. E. (1966). GERT: Graphical evaluation and review techniques. Technical report.

Guo, J. F., \& Qiao, L. H. (2004). Modeling of product development process with graphical evaluation and review technique. Computer Integrated Manufacturing Systems, 10, 758-763.

Gangeshwer, D. K., \& Shankar, G. (2015). GERT analysis of the deferred life test sampling plan based on gamma failure. International Journal of Quality Engineering and Technology, 5(3-4), 204-216.

Hayashi, A., Kosugi, T., \& Yoshida, H. (2005). Evaluation of polymer electrolyte fuel cell application technology R\&Ds by GERT analysis. International Journal of Hydrogen Energy, 30(9), 931-941.

Hu, M., Fang, Z., Liu, S., Chen, W., \& Yang, B. (2010, October). Study on evolution mechanism of fateful stampede accident based on graphical evaluation and review technique. In Systems Man and Cybernetics (SMC), 2010 IEEE International Conference on (pp. 2976-2978). IEEE.

Kosugi, T., Hayashi, A., Matsumoto, T., Akimoto, K., Tokimatsu, K., Yoshida, H., ... \& Kaya, Y. (2004). Time to realization: Evaluation of $\mathrm{CO}_{2}$ capture technology R\&Ds by GERT (Graphical Evaluation and Review Technique) analyses. Energy, 29(9), 1297-1308.

Li, C., Tang, Y., \& Li, C. (2011, August). A GERT-based analytical method for remanufacturing process routing. In Automation Science and Engineering (CASE), 2011 IEEE Conference on (pp. 462467). IEEE.

Li, C. C., Li, C. B., Cao, H. J., \& Yi, Q. (2012). Uncertain remanufacturing process routings model for used components based on GERT network. Computer Integrated Manufacturing Systems, 18(2), 298-305.

Li, C. (2014). An analytical method for cost analysis in multi-stage supply chains: a stochastic network model approach. Applied Mathematical Modelling, 38(11), 2819-2836.

Lin, K. P., Wu, M. J., Hung, K. C., \& Kuo, Y. (2011a). Developing a T $\omega$ (the weakest t-norm) fuzzy GERT for evaluating uncertain process reliability in semiconductor manufacturing. Applied soft computing, 11(8), 5165-5180.

Lin, K. P., Wen, W., Chou, C. C., Jen, C. H., \& Hung, K. C. (2011b). Applying fuzzy GERT with approximate fuzzy arithmetic based on the weakest t-norm operations to evaluate repairable reliability. Applied Mathematical Modelling, 35(11), 5314-5325.

Lin, K. P., Hung, K. C., Lai, S. P., Yu, Y. T., \& Wu, P. T. (2011c). Applying an approximate T $\omega$ (the weakest t-norm) fuzzy GERT to evaluate two-unit standby redundant system reliability. Procedia Computer Science, 4, 1326-1335.

Liu, X., Fang, Z., \& Zhang, N. (2017). A value transfer GERT network model for carbon fiber industry chain based on input-output table. Cluster Computing, 1-9.

Mohan, P., Agarwal, M., \& Sen, K. (2009, July). Reliability analysis of sparsely connected consecutivek systems: GERT approach. In Reliability, Maintainability and Safety, 2009. ICRMS 2009. 8th International Conference on (pp. 213-218). IEEE.

Nelson, R. G., Azaron, A., \& Aref, S. (2016). The use of a GERT based method to model concurrent product development processes. European Journal of Operational Research, 250(2), 566-578.

Ohta, T. (1978). Theoretical population genetics of repeated genes forming a multigene family. Genetics, 88(4), 845-861.

Pritsker, A. B., \& Whitehouse, G. E. (1966). GERT-Graphical evaluation and review techniques: Probabilistic and industrial engineering applications. Journal of Industrial Engineering, 17(6), 293. 
Sridharan, V., \& Kalyani, T. V. (2002). Stochastic analysis of a non-identical two-unit parallel system with common-cause failure using GERT technique. International journal of information and management sciences, 13(1), 49-57.

Tao, L., Wu, D., Liu, S., \& Lambert, J. H. (2017). Schedule Risk Analysis for New-Product Development: The GERT Method Extended by a Characteristic Function. Reliability Engineering \& System Safety.

Vaerst, A. (1980). Griechische Schildzeichen vom 8. bis zum ausgehenden 6. Jh (Doctoral dissertation, Universität Salzburg).

Wang, C. N., Yang, G. K., Hung, K. C., Chang, K. H., \& Chu, P. (2011). Evaluating the manufacturing capability of a lithographic area by using a novel vague GERT. Expert Systems with Applications, 38(1), 923-932.

Wu, Y., Pan, X., Kang, R., He, C., \& Gong, L. (2014). Multi-parameters uncertainty analysis of logistic support process based on GERT. Journal of Systems Engineering and Electronics, 25(6), 10111019.

Xu, Z., \& Jia, Z. J. (2010). Assessment of technical risk for weapon system development project based on GERT simulation including multi-branch feedback. Computer Integrated Manufacturing Systems, 16(3), 636-642.

Yamashiro, M. (2008). Analysis of network models using GERT for several kinds of probability distributions. Journal of Japan Industrial Management Association, 59(3), pp. 260-268.

Yi-song, Z., Dong, L., \& Feng, Z. (2009, October). Study on a GERT based method for hi-tech product development project planning. In Industrial Engineering and Engineering Management, 2009. IE\&EM'09. 16th International Conference on (pp. 1022-1026). IEEE.

Zhou, L., Xie, J., Gu, X., Lin, Y., Ieromonachou, P., \& Zhang, X. (2016). Forecasting return of used products for remanufacturing using Graphical Evaluation and Review Technique (GERT). International Journal of Production Economics, 181, 315-324.

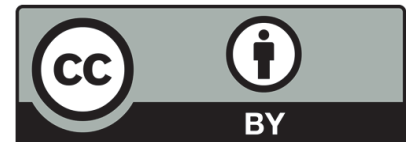

(C) 2017 by the authors; licensee Growing Science, Canada. This is an open access article distributed under the terms and conditions of the Creative Commons Attribution (CC-BY) license (http://creativecommons.org/licenses/by/4.0/). 